\title{
Recovery of high-pressure solid forms to ambient pressures
}

\author{
Martin R. Ward, Iain D. H. Oswald
}

Strathclyde Institute of Pharmacy and Biomedical Sciences (SIPBS), The University of Strathclyde, Glasgow, UK, martin.ward@strath.ac.uk

The discovery and study of new solid forms is a continual challenge in the field of solid-state science. The driving force behind this is the possibility that a new solid form of a given material might exhibit improved properties in comparison to the former e.g. increased solubility, improved mechanical properties. The use of high-pressure crystallographic methods has proven to be a highly efficient way to obtain new solid forms. ${ }^{1-3}$ Typically, these studies make use of a diamond anvil cell (DAC) to provide a high-pressure environment ( 0.1-100's GPa) with the sample crystal monitored by single crystal X-ray diffraction (SCXRD) during compression/decompression.

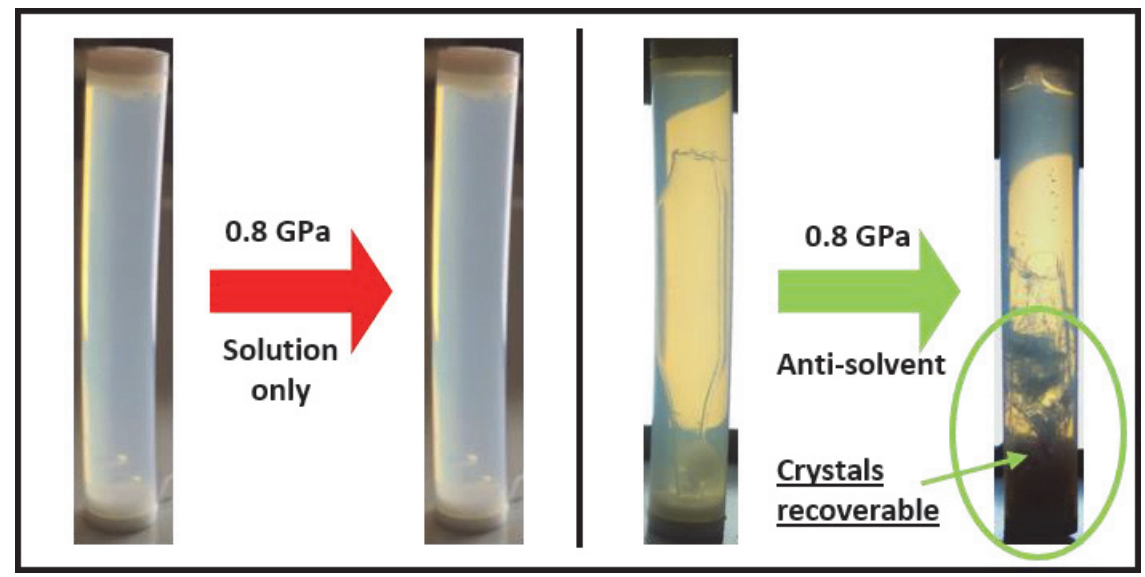

Figure 1. Illustration of anti-solvent addition process at high-pressure conditions

The main limitation of traditional high-pressure methods is that if a new solid form is discovered that is also recoverable to ambient pressure then only a small, single particle of the material is obtained. Harvesting a single particle does not allow for straightforward evaluation of important material properties such as flowability, rheology and thermal properties. Furthermore, using a single crystal to seed a crystallization process to scale up production is challenging.

In recent work, we have investigated the use of a large volume press to obtain high-pressure solid forms and successfully recover them back to ambient pressure in significant quantity (100-1000's mg) through compression or by anti-solvent addition at high pressure (approx. $0.8 \mathrm{GPa}$ ). Through these studies we have demonstrated the ability to obtain high pressure solid forms of para-aminobenzoic acid (delta polymorph), ${ }^{4}$ gamma-aminobutyric acid (GABA monohydrate), paracetamol (Orthorhombic, polymorph II) and maleic acid (polymorph II).

\section{References}

1 M. A. Neumann, J. van de Streek, F. P. A. Fabbiani, P. Hidber and O. Grassmann, Nat. Commun., 2015, 6, 7793.

2 R. D. L. Johnstone, A. R. Lennie, S. Parsons, E. Pidcock and J. E. Warren, Acta Crystallogr. Sect. BStructural Sci., 2009, 65, 731-748.

3 I. D. H. Oswald, I. Chataigner, S. Elphick, F. P. A. Fabbiani, A. R. Lennie, J. Maddaluno, W. G. Marshall, T. J. Prior, C. R. Pulham and R. I. Smith, CrystEngComm, 2009, 11, 359-366. M. R. Ward, S. Younis, A. J. Cruz-Cabeza, C. L. Bull, N. P. Funnell and I. D. H. Oswald, CrystEngComm, 2019, 21, 2058-2066. 